FOLIA

Amazónica

Revista del Instituto de Investigaciones

de la Amazonía Peruana

\title{
DIVERSIDAD Y COMPOSICIÓN FLORÍSTICA EN UN GRADIENTE ALTITUDINAL EN CHANCHAMAYO, SELVA CENTRAL DEL PERÚ
}

\author{
José GIACOMOTTI ${ }^{1}$, Carlos REYNEL ${ }^{1}$, Robin FERNANDEZ-HILARIO ${ }^{2,3}$, \\ Italo REVILLA ${ }^{4}$, Sonia PALACIOS-RAMOS ${ }^{1}$, Sara TERREROS-CAMAC ${ }^{1}$, \\ Aniceto DAZA ${ }^{1}$, Reynaldo LINARES-PALOMINO ${ }^{5}$
}

1 Herbario de la Facultad de Ciencias Forestales, Universidad Nacional Agraria La Molina. Lima, Perú.

2 División de Ecología Vegetal - CORBIDI. Lima, Perú.

3 Programa de Pós-Graduação em Botânica, Universidade Federal do Paraná. Curitiba, PR, Brazil.

${ }^{4}$ Instituto Científico Michael Owen Dillon - IMOD. Arequipa, Perú.

${ }^{5}$ Center for Conservation and Sustainability, Smithsonian Conservation Biology Institute. Lima, Perú.

* Correo electrónico: josegiacomottituezta@gmail.com

\section{RESUMEN}

Los bosques montanos y premontanos se extienden a lo largo de la Cordillera de los Andes, presentando diferentes ecosistemas y una gran diversidad de especies de flora y fauna silvestre. El objetivo de esta investigación fue evaluar la diversidad arbórea y la composición florística en seis parcelas permanentes de una hectárea, ubicadas en el estrato montano y premontano a lo largo de un gradiente altitudinal entre 800 y $3000 \mathrm{msnm}$, en la provincia de Chanchamayo, en la Selva Central del Perú. En la fase de campo se evaluaron todos los árboles con un DAP (diámetro a la altura de pecho) mayor o igual a $10 \mathrm{~cm}$. En las seis parcelas se registraron valores de 425 hasta 696 individuos por hectárea, y de 45 hasta 146 especies por hectárea. Los resultados de la composición florística en las parcelas localizadas en los bosques montanos (1500 a $3000 \mathrm{msnm}$ ), indicaron que las familias más importantes en general en términos de abundancia de individuos y número de especies fueron Lauraceae, Melastomataceae, Cunoniaceae y Rubiaceae. Mientras que, en las parcelas permanentes de los bosques premontanos ( 800 a $1500 \mathrm{msnm}$ ), las familias más importantes fueron Moraceae, Fabaceae y Lauraceae. Los resultados obtenidos muestran que la 
diversidad y composición florística de los bosques según el estrato altitudinal al que pertenecen.

PALABRAS CLAVE: Bosques montanos, comunidad vegetal, biodiversidad, conservación de bosques.

\title{
FLORISTIC DIVERSITY AND COMPOSITION ALONG AN ALTITUDINAL GRADIENT IN CHANCHAMAYO, FORESTS OF CENTRAL PERU
}

\begin{abstract}
The montane and premontane forests extend along the Andes mountains, presenting different ecosystems and a great diversity of species of wild flora and fauna. The objective of this research was to evaluate the tree diversity and the floristic composition in six permanent plots of one hectare, located in the montane and premontane strata along an altitudinal gradient between 800 and 3000 masl, in the province of Chanchamayo, in forests of Central Peru. In the field phase, all trees with a DBH (diameter at breast height) greater than or equal to $10 \mathrm{~cm}$ were evaluated. Values of 425 to 696 individuals per hectare, and of 45 to 146 species per hectare were recorded in the six plots. The results of the floristic composition in the plots located in the montane forests (1500 to 3000 masl), indicated that the most important families overall in terms of abundance of individuals and number of species were Lauraceae, Melastomataceae, Cunoniaceae and Rubiaceae. While, in the permanent plots of the premontane forests (800 to $1500 \mathrm{masl}$ ), the most important families were Moraceae, Fabaceae and Lauraceae. The results obtained show that the diversity and floristic composition change according to the altitudinal stratum to which they belong, changing the floristic composition of forests according to the altitudinal range where they are located.
\end{abstract}

KEYWORDS: Montane forests, plant community, biodiversity, forest conservation. 


\section{INTRODUCCIÓN}

Los bosques montanos en el neotrópico incluyen ecosistemas frágiles y de gran importancia, localizados en zonas de recarga de cuencas hidrográficas necesarias para el abastecimiento de agua, otorgando diferentes bienes y servicios ecosistémicos a las poblaciones de los Andes (Cuesta et al., 2009). En la región tropical, estos bosques presentan una elevada diversidad de especies de plantas, con una gran abundancia de formas de vida, junto con altas densidades de líquenes, musgos y plantas herbáceas (Tejedor Garavito et al., 2012), lo que hace necesario el estudio de la diversidad de su flora. Sin embargo, dado que estos bosques cubren rangos altitudinales en algunos casos de más de 2000 $\mathrm{m}$, se han descrito diferencias importantes entre zonas bajas, medias y altas de los rangos de ocurrencia y dominancia de familias botánicas (Young \& León, 2001; Arellano \& Macía, 2014), patrones estructurales y de biomasa vegetal sobre el suelo (Homeier et al., 2010; Girardin et al., 2014), entre otros. Por otro lado, hay evidencia que fragmentos de bosque montano localizados en montañas diferentes, pueden tener comunidades de plantas muy distintas (Wilson \& Rhemtulla, 2018).

Por estas características de los bosques montanos, es necesario seguir incrementando nuestro conocimiento de su composición florística a lo largo de gradientes altitudinales, y por lo tanto ecológicos, geográficos y/o climáticos (Malizia et al., 2020). Esto permitirá gestionarlos mejor identificando bosques o especies en riesgo (Dueñas \& Garate, 2018) y generando estrategias de conservación que lleven al aprovechamiento sostenible de sus recursos naturales.

Los bosques montanos y premontanos del Perú que se extienden a lo largo de la Cordillera de los Andes, se dividen por su rango altitudinal, mientras que el estrato premontano se extiende desde los 800 hasta los $1500 \mathrm{msnm}$, el estrato montano abarca desde los 1500 hasta los 3500 msnm (Antón \& Reynel, 2004). En el Perú estos bosquesseencuentran sobretierras deprotección, donde existe un mosaico de comunidades vegetales de variada composición, estructura y estado de conservación (Tovar et al., 2010). El presente trabajo se construyó sobre experiencias previas en parcelas permanentes de monitoreo de vegetación instaladas en bosques montanos y premontanos de Chanchamayo (Antón \& Reynel, 2004), y tiene por objetivo analizar y reportar la diversidad arbórea y la composición florística en una red de parcelas permanentes establecidas en la Selva Central del Perú.

\section{MATERIAL Y MÉTODOS}

\section{ÁREA DE ESTUDIO}

Este estudio se desarrolló en seis parcelas permanentes ubicadas en la provincia de Chanchamayo del departamento de Junín, a lo largo de un gradiente altitudinal entre los $800 \mathrm{y}$ $3000 \mathrm{msnm}$. Estas parcelas fueron remedidas para estudiar su diversidad arbórea y composición florística, y son parte del proyecto "Dinámica de los bosques de la Selva Central del Perú y su adecuación ante el Cambio Climático" (DINAFOR). Tres parcelas permanentes están ubicadas en los bosques de Puyu Sacha en Chanchamayo (Figura 1) en el estrato montano (entre 1500 a 3000 msnm), en la zona de vida denominada Bosque muy húmedo Montano Bajo Tropical (bmhMBT) (ONERN, 1976), y presentan las siguientes coordenadas geográficas: Puyu Sacha Montano Alto (451870 E y 8772222 N), Puyu Sacha Ribera (452187 E y 8773141 N) y Puyu Sacha Ladera 2 (453373 E y $8773935 \mathrm{~N}$ ). Las otras tres parcelas están localizadas en los bosques del Instituto de Desarrollo Regional Fundo La Génova de la Universidad Nacional Agraria La Molina en 


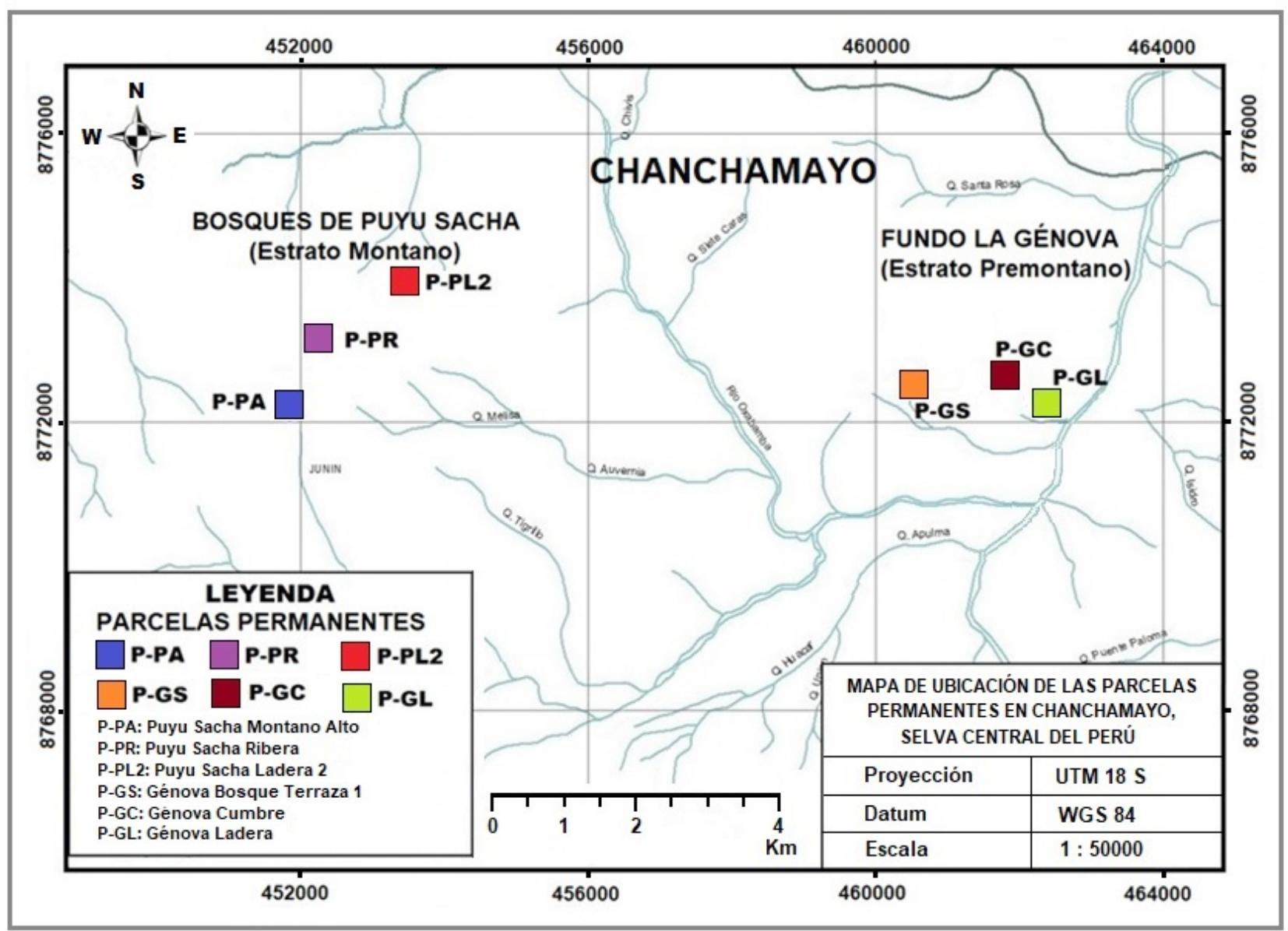

Figura 1: Mapa de ubicación de las seis parcelas permanentes en Chanchamayo, Selva Central del Perú.

Chanchamayo (Figura 1) dentro del estrato premontano (800 a $1500 \mathrm{msnm}$ ), en la zona de vida Bosque húmedo Premontano tropical (bhT) (ONERN, 1976), presentando las siguientes coordenadas geográficas: Génova Cumbre (461821 E y 8772580 N), Génova Bosque Terraza 1 (460545 E y 8772506 N) y Génova Ladera (461983 E y $8772505 \mathrm{~N}$ ).

\section{EVALUACION DE PARCELAS PERMANENTES}

Se realizó la remedición de seis parcelas permanentes de 1 hectárea, las cuales fueron establecidas y remedidas usando la metodología sugerida por RAINFOR (Phillips et al., 2016).
El trabajo de campo consistió en remedir a todos los individuos arbóreos y arborescentes que presentaron un diámetro a la altura de pecho (DAP) mayor o igual a $10 \mathrm{~cm}$. Todos los individuos de las parcelas fueron codificados y placados, realizando la identificación botánica de las especies en campo, y para aquellas que no fue posible la identificación, se realizaron colectas botánicas, las cuales fueron llevadas al Herbario de la Facultad de Ciencias Forestales de la Universidad Nacional Agraria La Molina (UNALM) para su identificación y almacenamiento. Seguimos al Angiosperm Phylogeny Group (APG IV, 2016) para asignar las especies y géneros a familias botánicas. Los nombres científicos de las especies fueron escritos deacuerdo con TROPICOS 
(www.tropicos.org). El permiso de colecta de las muestras botánicas corresponde a la Resolución de Dirección General № 147-2017-SERFORDGGSPFFS. Se sistematizó la información de todas las parcelas en una base de datos para determinar los parámetros de diversidad alfa y composición florística. Se usó el programa PAST (Hammer et al., 2001) para calcular el índice de diversidad de alfa Fisher y el software R Studio para realizar un análisis jerárquico entre las parcelas con distancia euclidiana usando el método Ward ( $R$ Core Team, 2018), obteniendo un dendrograma que indica el grado de afinidad florística a nivel de especies de los bosques estudiados.

\section{RESULTADOS Y DISCUSIÓN}

\section{ABUNDANCIA Y DIVERSIDAD}

En las seis parcelas estudiadas encontramos valores de abundancia con rangos que van desde 425 hasta 696 individuos por hectárea (Tabla 1). En cuanto al número de especies, reportamos valores de 45 hasta 146 especies por hectárea (Tabla 1). Estos valores son similares a los reportados en estudios previos en bosques montanos y premontanos en la selva central peruana (La Torre-Cuadros, 2003; Antón \& Reynel, 2004; Marcelo-Peña \& Reynel, 2014), y similares en valores de abundancia a los bosques de la Amazonía baja del Perú (Lewis et al., 2004; Dueñas \& Garate, 2018).

De los bosques montanos, P-PR y P-PL2, ubicados entre los 2000 hasta los $2300 \mathrm{msnm}$, presentaron un elevado número de especies (112 y 146 especies, respectivamente). En cambio, el bosque P-PA a $2770 \mathrm{msnm}$, reportó valores más bajos con 45 especies. Esta baja diversidad puede estar asociada a factores como la topografía, la altitud o el suelo. Pudiendo ser principalmente la altitud un factor limitante en la disminución del número de especies arbóreas para este bosque. A una determinada altitud por existir condiciones climáticas más adversas, pocas especies arbóreas podrían estar presentes. Por ejemplo, en bosques montanos de Bolivia, el número de especies registrados disminuyó conforme se aumentaba en altitud (Macía \& Fuertes, 2008).

El número de especies arbóreas varía entre las distintas parcelas estudiadas, y esta diversidad podría depender directamente de las condiciones particulares de cada bosque, como su propia vegetación, diferentes niveles de precipitación y composición de suelos (Gentry \& Ortiz,

Tabla 1: Abundancia y diversidad en las parcelas permanentes estudiadas en Chanchamayo, Selva Central del Perú.

\begin{tabular}{cccccccc}
\hline Parcela & $\begin{array}{c}\text { Altitud } \\
(\mathbf{m s n m})\end{array}$ & $\begin{array}{c}\text { Tipo de } \\
\text { bosque }\end{array}$ & $\begin{array}{c}\mathbf{N}^{\circ} \text { de } \\
\text { individuos }\end{array}$ & $\begin{array}{c}\mathbf{N}^{\circ} \text { de } \\
\text { especies }\end{array}$ & $\begin{array}{c}\mathbf{N}^{\circ} \text { de } \\
\text { géneros }\end{array}$ & $\begin{array}{c}\mathbf{N}^{\circ} \text { de } \\
\text { familias }\end{array}$ & $\begin{array}{c}\text { Índice de } \\
\text { Fisher }\end{array}$ \\
\hline P-PA & 2770 & Primario & 480 & 45 & 27 & 22 & 12,16 \\
P-PR & 2275 & Primario & 576 & 112 & 57 & 38 & 41,47 \\
P-PL2 & 2078 & Primario & 696 & 146 & 74 & 48 & 56,33 \\
P-GC & 1150 & Primario & 508 & 109 & 82 & 40 & 42,59 \\
P-GS & 1150 & Secundario & 512 & 70 & 52 & 32 & 21,93 \\
P-GL & 1075 & Primario & 425 & 72 & 55 & 28 & 24,87 \\
\hline
\end{tabular}

Parcelas en el estrato montano: Puyu Sacha Montano Alto (P-PA), Puyu Sacha Ribera (P-PR) y Puyu Sacha Ladera 2 (P-PL2). Parcelas en el estrato premontano: Génova Cumbre (P-GC), Génova Bosque Terraza 1 (P-GS) y Génova Ladera (P-GL). 
1993). La parcela P-GS ubicada en un bosque secundario de carácter transicional presentó un valor bajo de 70 especies, teniendo a Trophis caucana (82 individuos), Sapium glandulosum (67 individuos) y Mauria heterophylla (26 individuos) entre las principales especies que dominan su composición, las cuales han sido reportadas previamente como características de bosques secundarios de Chanchamayo (Reynel \& León, 1989; Echia et al., 2019). Si bien Trophis caucana y Sapium glandulosum son comunes en el valle de Chanchamayo, su abundancia es un indicador del estado sucesional de un bosque. Es relevante mencionar que los bosques del valle de Chanchamayo han sufrido un alto grado de intervención antrópica, principalmente a través de procesos de deforestación que han removido hasta $80 \%$ de la cubierta forestal nativa original (Reynel, 2012), reflejado en la disminución de los valores de riqueza de especies. La parcela P-GL localizada sobre un bosque primario, registró sólo 72 especies, esta baja riqueza puede estar asociada al bajo número de individuos que presenta (425 individuos, el valor más bajo entre todas las parcelas), y a que esta parcela se encuentra localizada en una zona de ladera, donde debido a las fuertes precipitaciones $y$ a la pendiente del terreno, es común una alta mortalidad, lo que podría incidir en una baja población de individuos y una menor diversidad de especies. Las variables de suelo, clima y topografía influyen en que existan diferencias entre la diversidad florística en distintos sitios (Loza et al., 2010).

Los valores del índice de diversidad alfa de Fisher van de 12,16 a 56,33 (Tabla 1). Se reportó que las tres parcelas con los valores más altos de este índice: P-PL2 $(56,33)$, P-GC $(42,52)$ y P-PR $(41,47)$, fue como consecuencia de presentar los niveles más altos en número de especies: P-PL2 (146 especies), P-GC (109) y P-PR (112). Este parece ser un patrón recurrente en bosques montanos y amazónicos. En Colombia, por ejemplo, también se registraron el mayor número de especies en las parcelas con los mayores índices de diversidad alfa de Fisher (Cano \& Stevenson, 2009; López \& Duque, 2009). Además, en la Amazonía baja del Perú se registraron los valores más altos en los índices alfa de Fisher en las parcelas con el mayor número de especies (Dueñas \& Garate, 2018).

\section{COMPOSICIÓN FLORÍSTICA A NIVEL DE FAMILIAS}

En los bosques montanos las familias que presentaron la mayor abundancia de individuos fueron: Lauraceae, Melastomataceae, Cunoniaceae, Cyatheaceae y Myrtaceae (Tabla 2), siendo familias dominantes en este tipo de bosque de la Selva Central del Perú (La Torre-Cuadros, 2003; Reynel \& Honorio, 2004; Vásquez et al., 2005). Teniendo a Lauraceae y Melastomataceae como abundantes en diferentes bosques del estrato montano (Gentry, 1992), como en Cajamarca (Pérez, 2011) y Cusco (Rivera, 2007).

Se registró a Lauraceae, Melastomataceae, Rubiaceae y Myrtaceae como las familias con el mayor número de especies en los bosques montanos estudiados (Tabla 2). De estas familias, Melastomataceae y Rubiaceae se reportaron entre las seis familias de plantas con flores con mayor diversidad en bosques montanos neotropicales en el rango de 1500 a 3500 msnm (Young \& León, 2001). Además, Melastomataceae, Lauraceae y Rubiaceae estuvieron entre las familias con mayor número de especies en bosques montanos de áreas naturales protegidas de Oxapampa (Vásquez et al., 2005) y de Cajamarca (Pérez, 2011). Esto indicaría la amplia distribución, abundancia y diversidad de estas familias en bosques del estrato montano.

En los bosques premontanos la composición florística a nivel de familias presentó a Moraceae, Fabaceae, Euphorbiaceae, Lauraceae 
Tabla 2: Composición florística de las familias en los Bosques Montanos de Chanchamayo, Selva Central del Perú.

\begin{tabular}{lccccc}
\hline \multicolumn{7}{c}{ Familias con mayor abundancia en Bosques Montanos } \\
\hline \multicolumn{1}{c}{ Familia } & P-PA & P-PR & P-PL2 & Total individuos & \% del Total \\
\hline Lauraceae & 33 & 24 & 200 & 257 & 14,7 \\
Melastomataceae & 96 & 73 & 22 & 191 & 10,9 \\
Cunoniaceae & 108 & 82 & - & 190 & 10,8 \\
Cyatheaceae & 48 & 83 & - & 131 & 7,5 \\
Myrtaceae & 3 & 24 & 64 & 91 & 5,2 \\
Resto de familias & 192 & 290 & 410 & 892 & 50,9 \\
\hline Total individuos & 480 & 576 & 696 & 1752 & 100 \\
\hline \multicolumn{1}{c}{ Familia } & Familias con mayor número de especies en Bosques Montanos & \\
\hline Lauraceae & P-PA & P-PR & P-PL2 & Total especies & $\%$ del Total \\
Melastomataceae & 4 & 3 & 17 & 23 & 9,1 \\
Rubiaceae & 8 & 11 & 6 & 22 & 8,7 \\
Myrtaceae & 2 & 4 & 9 & 13 & 5,1 \\
Resto de familias & 1 & 2 & 9 & 11 & 4,3 \\
\hline Total especies & 30 & 92 & 105 & 184 & 72,7 \\
\hline
\end{tabular}

Tabla 3: Composición florística de las familias en los Bosques Premontanos en Chanchamayo, Selva Central del Perú.

\begin{tabular}{lccccc}
\hline \multicolumn{5}{c}{ Familias con mayor abundancia en Bosques Premontanos } & \\
\hline Familia & P-GC & P-GS & P-GL & Total individuos & \% del Total \\
\hline Moraceae & 118 & 153 & 118 & 389 & 26,9 \\
Fabaceae & 62 & 30 & 22 & 114 & 7,9 \\
Euphorbiaceae & 19 & 88 & 4 & 111 & 7,7 \\
Lauraceae & 34 & 35 & 38 & 107 & 7,4 \\
Urticaceae & 24 & 41 & 23 & 88 & 6,1 \\
Resto de familias & 251 & 165 & 220 & 636 & 44,0 \\
\hline Total individuos & 508 & 512 & 425 & 1445 & 100 \\
\hline \multicolumn{1}{c}{ Familia } & Familias con mayor número de especies en Bosques Premontanos & \\
\hline Fabaceae & P-GC & P-GS & P-GL & Total especies & $\%$ del Total \\
Moraceae & 13 & 8 & 7 & 20 & 11,7 \\
Lauraceae & 10 & 10 & 11 & 18 & 10,5 \\
Rubiaceae & 13 & 5 & 6 & 17 & 9,9 \\
Malvaceae & 9 & 2 & 2 & 9 & 5,3 \\
Urticaceae & 4 & 4 & 6 & 8 & 4,7 \\
Resto de familias & 6 & 3 & 3 & 8 & 4,7 \\
\hline Total especies & 54 & 38 & 37 & 91 & 53,2 \\
\hline
\end{tabular}


Tabla 4: Composición florística de los géneros en los Bosques Montanos en Chanchamayo, Selva Central del Perú.

\begin{tabular}{|c|c|c|c|c|c|}
\hline \multicolumn{6}{|c|}{ Géneros con mayor abundancia en Bosques Montanos } \\
\hline & P-PA & P-PR & P-PL2 & Total individuos & $\%$ del Total \\
\hline Weinmannia & 108 & 82 & - & 190 & 10,8 \\
\hline Miconia & 94 & 54 & 18 & 166 & 9,5 \\
\hline Hieronyma & 16 & 25 & 38 & 79 & 4,5 \\
\hline Nectandra & - & 16 & 57 & 73 & 4,2 \\
\hline Alsophila & - & 72 & - & 72 & 4,1 \\
\hline Persea & - & - & 72 & 72 & 4,1 \\
\hline Resto de géneros & 262 & 327 & 511 & 1100 & 62,8 \\
\hline Total individuos & 480 & 576 & 696 & 1752 & 100 \\
\hline \multicolumn{6}{|c|}{ Géneros con mayor número de especies en Bosques Montanos } \\
\hline Género & P-PA & P-PR & P-PL2 & Total especies & $\%$ del Total \\
\hline Miconia & 7 & 8 & 5 & 17 & 6,7 \\
\hline Inga & - & 2 & 6 & 8 & 3,2 \\
\hline Myrcia & 1 & - & 6 & 7 & 2,8 \\
\hline Ocotea & 3 & 1 & 3 & 7 & 2,8 \\
\hline Ficus & - & 2 & 6 & 6 & 2,4 \\
\hline Nectandra & - & 2 & 5 & 6 & 2,4 \\
\hline Resto de géneros & 34 & 97 & 115 & 202 & 79,8 \\
\hline Total especies & 45 & 112 & 146 & 253 & 100 \\
\hline
\end{tabular}

y Urticaceae como las de mayor abundancia de individuos (Tabla 3). Mientras que, Fabaceae, Moraceae, Lauraceae, Rubiaceae, Malvaceae y Urticaceae fueron las que presentaron el mayor número de especies (Tabla 3). Dentro de estas, se menciona a Fabaceae, Moraceae y Rubiaceae como dominantes entre los 1000 a 1500 msnm (Young \& León, 2001). En base a los resultados, las tres familias más importantes en este estudio fueron Moraceae, Fabaceae y Lauraceae, las cuales son reportadas con un amplio número de individuos y especies en bosques premontanos de Chanchamayo (Phillips \& Miller, 2002; Antón \& Reynel, 2004; Cotito, 2014; Echia et al., 2019) y Satipo (Rivera, 2014), de estas tres familias, destacamos a Lauraceae por registrar altos niveles de individuos y especies en los estratos montano y premontano estudiados.

\section{COMPOSICIÓN FLORÍSTICA A NIVEL DE} GÉNEROS

En los bosques montanos los géneros con la mayor cantidad de individuos fueron Weinmannia, Miconia, Hieronyma, Nectandra, Alsophila y Persea (Tabla 4). De estos géneros, se reportaron como abundantes a Weinmannia en un bosque montano del Cusco (Rivera, 2007), y a Miconia junto a Nectandra en un área de ladera del estrato montano en Chanchamayo (Reynel \& Honorio, 2004). En cuanto a los géneros con mayor número de especies, estos fueron Miconia, Inga, Myrcia, Ocotea, Ficus y Nectandra (Tabla $4)$, los cuales ya han sido registrados con varias especies en bosques montanos del Perú (Young \& León, 1999). En la selva central destacamos a Miconia, Ficus, Nectandra y Ocotea entre los de mayor número de especies en bosques montanos de Chanchamayo (La Torre-Cuadros, 2003; 
Tabla 5: Composición florística de los géneros en los Bosques Premontanos en Chanchamayo, Selva Central del Perú.

\begin{tabular}{|c|c|c|c|c|c|}
\hline \multicolumn{6}{|c|}{ Géneros con mayor abundancia en Bosques Premontanos } \\
\hline Género & P-GC & P-GS & P-GL & Total individuos & $\%$ del Total \\
\hline Trophis & 26 & 82 & 23 & 131 & 9,1 \\
\hline Sapium & 17 & 67 & - & 84 & 5,8 \\
\hline Nectandra & 21 & 31 & 30 & 82 & 5,7 \\
\hline Inga & 42 & 17 & 19 & 78 & 5,4 \\
\hline Ficus & 5 & 62 & 6 & 73 & 5,1 \\
\hline Resto de géneros & 397 & 253 & 347 & 997 & 69,0 \\
\hline Total individuos & 508 & 512 & 425 & 1445 & 100 \\
\hline \multicolumn{6}{|c|}{ Géneros con mayor número de especies en Bosques Premontanos } \\
\hline Género & P-GC & P-GS & P-GL & Total especies & $\%$ del Total \\
\hline Inga & 7 & 4 & 4 & 10 & 5,8 \\
\hline Ficus & 3 & 7 & 4 & 9 & 5,3 \\
\hline Allophylus & 2 & 2 & 1 & 4 & 2,3 \\
\hline Eugenia & 2 & 2 & - & 4 & 2,3 \\
\hline Nectandra & 3 & 2 & 2 & 4 & 2,3 \\
\hline Ocotea & 4 & 1 & 2 & 4 & 2,3 \\
\hline Resto de géneros & 88 & 52 & 59 & 136 & 79,5 \\
\hline Total especies & 109 & 70 & 72 & 171 & 100 \\
\hline
\end{tabular}

Reynel \& Honorio, 2004) y de Oxapampa (Gómez, 2000). Se puede indicar que la presencia de géneros como Weinmannia y Miconia diferencia al estrato montano del premontano (MarceloPeña \& Reynel, 2014), teniendo ambos una mayor diversidad en bosques montanos andinos.

En los bosques premontanos abundaron individuos de un set de géneros muy diferente, siendo Trophis, Sapium, Nectandra, Inga y Ficus los géneros con mayor abundancia de individuos (Tabla 5). De estos, tenemos a Inga y Ficus, con 10 y 9 especies respectivamente, como los dos géneros más especiosos en el estrato premontano, seguidos de Allophylus, Eugenia, Nectandra y Ocotea con cuatro especies cada uno (Tabla 5). Ficus y Ocotea fueron mencionadas como géneros con amplia diversidad en la selva central (Marcelo-Peña \& Reynel, 2014). En los bosques de Chanchamayo se ha reportado una numerosa cantidad de especies para los géneros Ficus (Cáceres \& Reynel, 2010) e Inga (Reynel \& Pennington, 1997), teniendo Inga una amplia presencia y riqueza de especies en el estrato premontano (Estrada, 2007). En esta investigación, Ficus, Inga, Ocotea y Nectandra se registraron entre los géneros más especiosos en los bosques montanos y premontanos estudiados, teniendo Nectandra altos niveles de abundancia en ambos estratos, esto indicaría la importancia ecológica de estos cuatro géneros en la Selva Central de Perú.

\section{ANÁLISIS DE AGRUPAMIENTO A NIVEL DE ESPECIES}

El análisis de agrupamiento basado en la composición florística a nivel de especies de las seis parcelas estudiadas se representó en un dendrograma con dos grupos bien definidos: 


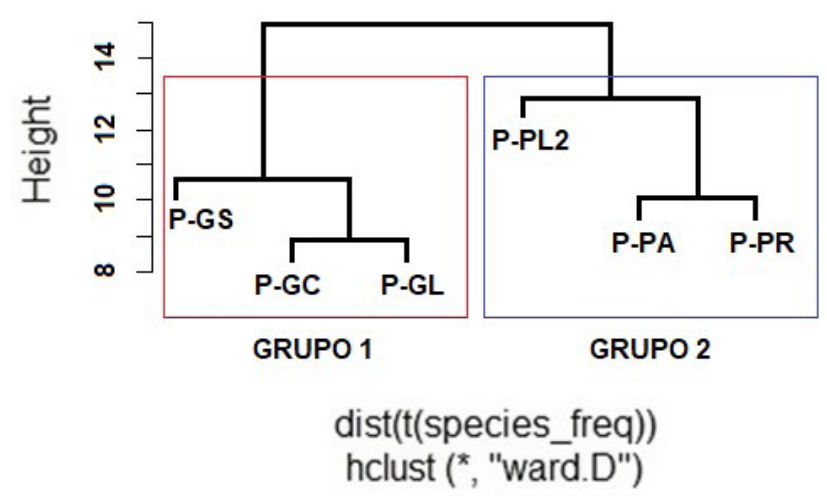

Figura 2: Dendrograma de agrupamiento a nivel de especies en seis parcelas permanentes en Chanchamayo, Selva Central del Perú.

Grupo 1 y Grupo 2 (Figura 2), en los cuales hay una tendencia de agrupamiento principalmente por el estrato altitudinal donde se encuentran estas parcelas.

El Grupo 1 está integrado por las parcelas P-GS, P-GC y P-GL, cuya característica principal es que se encuentran localizadas dentro del estrato premontano del Fundo La Génova. Estas parcelas están distanciadas unos pocos kilómetros entre sí, estando P-GS y P-GC a una altitud de $1150 \mathrm{msnm}$, mientras que P-GL a 1075 msnm. Dentro de este grupo vemos que las parcelas presentan mayor similitud por el tipo de bosque donde se encuentran, como es el caso de P-GC y P-GL localizadas en bosques primarios, presentando afinidad florística de especies propias de vegetación primaria como Batocarpus costaricensis, Nectandra pulverulenta, Pseudolmedia laevis y Otoba parvifolia (Reynel et al., 2003; Quintero, 2020). En cambio, la parcela P-GS es menos afín a las dos primeras porque se encuentra localizada en un bosque secundario y por ende, contiene especies como Cecropia polystachya (17 individuos), Ficus insipida (16 individuos) y Piper aduncum (15 individuos), representativas de la vegetación secundaria de la zona (Reynel \& León, 1989; Cáceres, 2005). Esto indicaría que dentro del Grupo 1 las parcelas se agrupan por el tipo de vegetación que contienen. De las 171 especies forestales distribuidas entre las parcelas del Grupo 1, fueron 17 especies las que se repitieron de forma simultánea en estas tres parcelas, destacando ente las más abundantes: Trophis caucana (131 individuos), Nectandra pulverulenta (77 individuos), Clarisia biflora (52 individuos), Mauria heterophylla (37 individuos) e Inga cinnamomea (33 individuos), las cuales son características de bosques premontanos de Chanchamayo (Antón \& Reynel, 2004; Cotito, 2014; Echia et al., 2019).

El Grupo 2 está formado por las parcelas P-PA, P-PR y P-PL2 pertenecientes al estrato montano (1500 a $3500 \mathrm{msnm}$ ) y ubicadas dentro del bosque de Puyu Sacha en Chanchamayo. En este grupo se puede observar que a pesar de que P-PA se ubica a 2770 msnm y P-PR a 2275 msnm, estas dos parcelas presentaron la mayor similitud debido a que ambas se localizan en bosques primarios con un alto estado de conservación, teniendo una mayor afinidad florística a nivel de especies. En cambio, debido a que P-PL2 se localiza en un bosque donde hubo perturbaciones antropogénicas en el pasado (Llacsahuanga, 2015), presentó menor similitud respecto a P-PA y P-PR. Dentro del Grupo 2 las parcelas se agrupan por el tipo de vegetación, en este caso aparentemente son más similares los bosques conservados. Las parcelas P-PA y P-PR compartieron nueve especies: Hieronyma macrocarpa (39 individuos), Myrsine coriacea (21 individuos), Gordonia fruticosa (19 individuos), Miconia aff. crassistigma (19 individuos), Miconia sp.2 (6 individuos), Hedyosmum racemosum (5 individuos), Miconia sp.10 (5 individuos), Brunellia sp.1 (3 individuos) y Hieronyma oblonga (2 individuos). Mientras que, de las 253 especies forestales distribuidas entre estos bosques montanos, solo dos especies ocurren en las tres parcelas: Hieronyma oblonga (40 individuos) y Gordonia fruticosa (20 individuos). 
En el dendrograma obtenido se muestra que el grado de afinidad por especies de las seis parcelas (Figura 2) está marcado en primer lugar por el estrato altitudinal, y en menor medida, por el tipo de bosque donde estas se encuentran.

\section{CONCLUSIONES}

Los bosques del estrato premontano y montano cambian su composición florística a lo largo del gradiente altitudinal, y cuando tuvieron intervención antrópica previa como el caso de P-GS y P-PL2, presentando especies, géneros y familias características.

La presencia de familias como Moraceae, Fabaceae y Lauraceae en el estrato premontano, y de Melastomataceae, Cunoniaceae y Cyatheaceae en el estrato montano, nos ayudan a caracterizar ambos estratos, como se ha reportado en este estudio y en otras investigaciones realizadas en la Selva Central del Perú.

Es necesaria la instalación y remedición de parcelas permanentes para complementar los estudios sobre diversidad y composición florística en gradientes de elevación y zonas con perturbaciones antrópicas en la Selva Central del Perú, y analizarlos junto a otros estudios de bosques montanos andinos.

\section{AGRADECIMIENTOS}

Esta investigación fue financiada por Cienciactiva, CONCYTEC.

\section{REFERENCIAS BIBLIOGRÁFICAS}

Antón, D.; Reynel, C. 2004. Relictos de bosques de excepcional diversidad en los Andes Centrales del Perú. Herbario de la Facultad de Ciencias
Forestales de la Universidad Nacional Agraria La Molina, Lima. 323pp.

APG IV. 2016. An update of the Angiosperm Phylogeny Group classification for the orders and families of flowering plants: APG IV. Botanical Journal of the Linnean Society, 181: 1-20. DOI: https://doi.org/10.1111/ boj.12385

Arellano, G.; Macía, M.J. 2014. Local and regional dominance of woody plants along an elevational gradient in a tropical montane forest of northwestern Bolivia. Plant Ecology, 215: 39-54. DOI: https://doi.org/10.1007/ s11258-013-0277-8

Cáceres, B. 2005. Diversidad de la composición florística de la microcuenca de Santa Rosa, Chanchamayo, Junín. Tesis de maestría, Universidad Nacional Agraria La Molina, Lima, Perú. 241pp.

Cáceres, P.; Reynel, C. 2010. Los árboles de Ficus (Ojé) del valle de Chanchamayo, Dpto. de Junín, Perú (800-2500 msnm). APRODES, Lima. 79pp.

Cano, A.; Stevenson, P.R. 2009. Diversidad y composición florística de tres tipos de bosque en la Estación Biológica Caparú, Vaupés. Revista Colombiana Forestal, 12: 63-80. DOI: https://doi.org/10.14483/udistrital.jour. colomb.for.2009.1.a06

Cotito, S. 2014. Diversidad y composición florística de un área de bosque ribereño premontano del Valle de Chanchamayo. Tesis de maestría, Universidad Nacional Agraria La Molina, Lima, Perú. 128pp.

Cuesta, F.; Peralvo, M.; Valarezo, N. 2009. Los Bosques Montanos de los Andes Tropicales. CONDESAN, Quito. 73pp.

Dueñas, H.; Garate, J.S. 2018. Diversidad, dominancia y distribución arbórea en Madre de Dios, Perú. Revista Forestal del Perú, 33(1): 4-23. DOI: http://dx.doi.org/10.21704/rfp. v33i1.1152 
Echia, E.; Reynel, C.; Manta, M. 2019. La flora leñosa establecida luego de las quemas en el valle de Chanchamayo - Selva Central del Perú. Revista Forestal del Perú, 34(1): 83-101. DOI: http://dx.doi.org/10.21704/rfp.v34i1.1287

Estrada, Z. 2007. Análisis de interpretación de la diversidad florística en Bosques Húmedos del Perú, con énfasis al estudio del "Bosque Macuya" del distrito de Irazola, provincia de Padre Abad, departamento de Ucayali. Tesis de doctorado, Universidad Nacional Mayor de San Marcos, Lima, Perú. 182pp.

Gentry, A.H. 1992. Diversity and floristic composition of Andean forest of Peru and adjacent countries: implications for their conservation. En: Young, K.R.; Valencia, N. (Eds). Biogeografía, Ecología y Conservación del Bosque Montano en el Perú. p.11-29.

Gentry, A.H; Ortiz, R. 1993. Patrones de composición florística en la Amazonía peruana. En: Kalliola, R.; Puhakka, M.; Danjoy, W. (Eds). Amazonía peruana, vegetación húmeda tropical en el llano subandino. p.155-166.

Girardin, C.A.J.; Farfan-Rios, W.; Garcia, K.; Feeley, K.J.; Jørgensen, P.M.; Araujo-Murakami, A.; Cayola-Pérez, L.; Seidel, R.; Paniagua, N.; Fuentes Claros, A.F.; Maldonado, C.; Silman, M.; Salinas, N.; Reynel, C.; Neill, D.A.; Serrano, M.; Caballero, C.J.; La Torre-Cuadros, M.A.; Macía, M.J; Killeen, T.J.; Malhi, Y. 2014. Spatial patterns of above-ground structure, biomass and composition in a network of six Andean elevation transects. Plant Ecology \& Diversity, 7(1-2): 161-171. DOI: https://doi.org/10.108 0/17550874.2013.820806

Gómez, D. 2000. Composición florística en el bosque ribereño de la Cuenca alta San Alberto, Oxapampa - Perú. Tesis de pre-grado, Universidad Nacional Agraria La Molina, Facultad de Ciencias Forestales, Lima, Perú. 177pp.
Hammer, Ø.; Harper, D.A.T.; Ryan, P.D. 2001. PAST: Paleontological statistics software package for education and data analysis. Palaeontologia Electronica, 4(1): 1-9.

Homeier, J.; Breckle, S.W.; Günter, S.; Rollenbeck, R.T.; Leuschner, C. 2010. Tree diversity, forest structure and productivity along altitudinal and topographical gradients in a species-rich ecuadorian montane rain forest. Biotropica, 42(2): 140-148. DOI: https://doi. org/10.1111/j.1744-7429.2009.00547.x

La Torre-Cuadros, M.A. 2003. Composición florística y diversidad en el bosque relicto los Cedros de Pampa Hermosa (Chanchamayo, Junín) e implicancias para su conservación. Tesis de maestría, Universidad Nacional Agraria La Molina, Lima, Perú. 141pp.

Lewis, S.L.; Phillips, O.L.; Baker, T.S.; Lloyd, J.; Malhi, Y.; Almeida, S.; Higuchi, N.; Laurance, W.F.; Neill, D.A.; Silva, J.N.M.; Terborgh, J.; Torrez Lezama, A.; Vásquez, R.; Brown, S.; Chave, J.; Kuebler, C.; Nuñez Vargas, P.; Vinceti, B. 2004. Concerted changes in tropical forest structure and dynamics: evidence from 50 South American long-term plots. Philosophical Transactions of the Royal Society London B, 359: 421-436. DOI: https://doi.org/10.1098/ rstb.2003.1431

López, W.; Duque, A. 2009. Patrones de diversidad alfa en tres fragmentos de bosques montanos en la región norte de los Andes, Colombia. Revista de Biología Tropical, 58(1): 483498. DOI: https://doi.org/10.15517/rbt. v58i1.5223

Loza, I.; Moraes, M.; Jørgensen, P.M. 2010. Variación de la diversidad y composición florística en relación a la elevación en un bosque montano boliviano (PNANMI Madidi). Ecología en Bolivia, 45(2): 87-100.

Llacsahuanga, J. 2015. Composición y diversidad arbórea de un área en un bosque montano 
nublado en Puyu Sacha, Chanchamayo, Junín. Tesis de pre-grado, Universidad Nacional Agraria La Molina, Facultad de Ciencias Forestales, Lima, Perú. 64pp.

Macía, M.; Fuertes, J. 2008. Composición florística y estructura de los árboles en un bosque tropical montano de la Cordillera Mosetenes, Bolivia. Revista Boliviana de Ecología y Conservación Ambiental, 23: 1-14.

Malizia, A.; Blundo, C.; Carilla, J.; Osinaga Acosta, O.; Cuesta, F.; Duque, A.; Aguirre, N.; Aguirre, Z.; Ataroff, M.; Baez, S.; Calderón-Loor, M.; Cayola, L.; Cayuela, L.; Ceballos, S.; Cedillo, H.; Farfan-Rios, W.; Feeley, K.J.; Fuentes, A.F.; Gámez Álvarez, L.E.; Grau, R.; Homeier, J.; Jadan, O.; Llambi, L.D.; Loza Rivera, M.I.; Macía, M.J.; Malhi, Y.; Malizia, L.; Peralvo, M.; Pinto, E.; Tello, S.; Silman, M.; Young, K.R. 2020. Elevation and latitude drives structure and tree species composition in Andean forests: Results from a large-scale plot network. PLOS ONE, 15(4): e0231553. DOI: https://doi.org/10.1371/ journal.pone.0231553

Marcelo-Peña, J.L.; Reynel, C. 2014. Patrones de diversidad y composición florística de parcelas de evaluación permanente en la selva central de Perú. Rodriguésia, 65(1): 3547. DOI: https://doi.org/10.1590/S217578602014000100003

ONERN. 1976. Mapa ecológico del Perú. Guía explicativa. Oficina Nacional de Evaluación de Recursos Naturales del Perú - ONERN, Lima. 146pp.

Pérez, G. 2011. Composición y diversidad de la flora arbórea en dos áreas de bosque en Huamantanga - Jaén - Cajamarca, Perú. Tesis de maestría, Universidad Nacional Agraria La Molina, Lima, Perú. 188pp.

Phillips, O.L.; Baker, T.R.; Feldpausch, T.; Brienen, R. 2016. Manual de campo para el establecimiento y la remedición de parcelas. RAINFOR. 28pp.
Phillips, O.L.; Miller, J. 2002. Global patterns of plant diversity: Alwyn H. Gentry's forest transect data set. Monographs in systematic Botany (MSB). Missouri Botanical Garden Press, Missouri. 319pp.

Quintero, F.; Cáceres, B.; Reynel, C.; FernandezHilario, R.; Wong Sato, A.A.; Chávez, J.; PalaciosRamos, S. 2020. Tiempos de recomposición de la diversidad arbórea a lo largo de la sucesión vegetal en los bosques del Valle de Chanchamayo / Junín / Perú. Ecología Aplicada, 19(2): 111-120. DOI: http://dx.doi. org/10.21704/rea.v19i2.1562

R Core Team. 2018. R: Alanguage and environment for statistical computing. R Foundation for Statistical Computing. (https://www.Rproject.org). Acceso: 29/10/2020.

Reynel, C.; León, J. 1989. Especies forestales de los bosques secundarios de Chanchamayo (Perú). UNALM, Lima. 173pp.

Reynel, C.; Pennington, R.T.; Pennington, T.D.; Flores, C.; Daza, A. 2003. Árboles útiles de la Amazonía Peruana: un manual con apuntes de identificación, ecología y propagación de las especies. Darwin Initiative Project. 509pp.

Reynel, C.; Honorio, E. 2004. Diversidad y composición de la flora arbórea en un área de ladera de bosque montano: Pichita, Valle de Chanchamayo, 2000 -2500 msnm. En: Antón, D.; Reynel. C. (Eds). Relictos de bosques de excepcional diversidad en los Andes Centrales del Perú. p. 45-98.

Reynel, C.; Pennington, T.D. 1997. El género Inga en el Perú. Morfología, distribución y usos. Royal Botanic Gardens, Kew, UK. 228pp.

Reynel, C. 2012. Contexto físico y biológico del Bosque Puyu Sacha. En: Reynel, C. (Ed). Flora y fauna del bosque montano nublado Puyu Sacha. p.5-13.

Rivera, G. 2007. Composición florística y análisis de diversidad arbórea en un área de bosque montano en el Centro de Investigación 
Wayqecha, Kosñipata Cusco. Tesis de pregrado, Universidad Nacional Agraria La Molina, Facultad de Ciencias Forestales, Lima, Perú. 84pp.

Rivera, R. 2014. Diversidad y composición florística en un área de bosque premontano, Fundo Santa Teresa, Río Negro, Satipo, Junín. Tesis de maestría, Universidad Nacional Agraria La Molina, Lima, Perú. 118pp.

Tejedor Garavito, N.; Álvarez, E.; Arango Caro, S.; Araujo Murakami, A.; Blundo, C.; Boza Espinosa, T.E.; La Torre-Cuadros, M.A.; Gaviria, J.; Gutiérrez, N.; Jørgensen, P.M.; León, B.; López Camacho, R.; Malizia, L.; Millán, B.; Moraes, M.; Pacheco, S.; Rey Benayas, J.M.; Reynel, C.; Timaná de la Flor, M.; Ulloa Ulloa, C.; Vacas Cruz, O.; Newton, A.C. 2012. Evaluación del estado de conservación de los bosques montanos en los Andes tropicales. Ecosistemas, 21(1-2): 148-166.

Tovar, A.; Tovar, C.; Saito, J.; Soto, A.; Regal, F.; Cruz, Z.; Véliz, C.; Vásquez, P.; Rivera, G. 2010. Yungas peruanas - Bosques montanos de la vertiente oriental de los Andes del Perú: Una perspectiva ecorregional de conservación. CDC-UNALM, Lima. 150pp.

Vásquez, R.; Rojas, R.; Monteagudo, A.; Meza, K.; Van Der Werff, H.; Ortiz-Gentry, R.; Catchpole, D. 2005. Flora vascular de la selva central del Perú: una aproximación de la composición florística de tres Áreas Naturales Protegidas. Arnaldoa, 12(1-2): 112-125.

Wilson, S.J.; Rhemtulla, J.M. 2018. Small montane cloud forest fragments are important for conserving tree diversity in the Ecuadorian Andes. Biotropica, 50(4): 586-597. DOI: https://doi.org/10.1111/btp.12542

Young, K.R.; León, B. 1999. Peru's humid eastern montane forests: An overview of their physical settings, biological diversity, human use and settlement, and conservation needs. Centre for Research on the Cultural and Biological Diversity of Andean Rainforests (DIVA), Kalø, Denmark. 97pp.

Young, K.R.; León, B. 2001. Perú. In: Kappelle, M.; Brown, A.D. (Eds). Bosques nublados del Neotrópico. p.549-580.

Recibido: 16 de marzo de 2021 Aceptado para publicación: 6 de julio de 2021 